\title{
Joint Tests of Linkage and Association for Quantitative Traits ${ }^{1}$
}

\author{
David B. Allison \\ Department of Biostatistics Section on Statistical Genetics \& Clinical Nutrition Research Center, \\ University of Alabama at Birmingham, Birmingham, Alabama 35294
}

and

\section{Michael C. Neale}

Department of Psychiatry, Medical College of Virginia, Virginia Commonwealth

University, Richmond, Virginia 23284

Received May 12, 2001

In this paper we describe various study designs and analytic techniques for testing the joint hypothesis that a genetic marker is both linked to and associated with a quantitative phenotype. Issues of power and sampling are addressed. The distinction between methods that explicitly examine association and those that infer association by examining the distribution of allelic transmissions from a heterozygous parent is examined. Extensions to multivariate, multiallelic, and multilocus situations are addressed. Recent approaches that combine variance-components-based linkage analyses with joint tests of linkage in the presence of association for disentanglement of the linkage and association and the application of such methods to fine mapping are discussed. Finally, new classes of joint tests of linkage and association that do not require samples of related individuals are described. ๑ 2001 Elsevier Science

\section{INTRODUCTION}

\section{The Distinction between Linkage And Association}

The overwhelming majority of inferential statistics involve tests of associations (Woodward et al., 1990). Even though research designs can appropriately be distinguished as experimental and correlational (Campbell and Stanley, 1966), even experimental studies ultimately test for association. Specifically, they test whether there is an association between the experimental condition cases are assigned to and the dependent variable. Similarly, although in genetics a distinction is made between linkage and association studies, it is important to

\footnotetext{
${ }^{1}$ This work was supported in part by NIH Grants P30DK056336, R01DK56366, K02MH001458, and R01ES09912.
}

realize that both ultimately test for association in both a statistical and a genetic sense (Elston, 1998, 2000). The socalled association studies test for the statistical significance of the sample association between genotype at a given locus and a phenotype as a means of inferring an association between the genotype and phenotype in the overall population. In contrast, linkage studies for quantitative traits usually test for the statistical significance of the association of functions of the number of alleles shared identical by descent (IBD) at a genetic locus among pairs of relatives and the degree of phenotypic similarity among these relatives (Lander and Schork, 1994). A statistically significant association implies that there is cosegregation of alleles at that locus and the phenotype within families in the overall population of families. This in turn implies that the locus under study is physically linked to (or is itself) a locus at which allelic variation causes variation in the phenotype. 


\section{Association Studies and Confounding}

Association studies can be confounded by population stratification. This is an example of what is referred to as "Simpson's paradox" (Simpson, 1951) and is illustrated in Fig. 1. If groups of individuals within a species form specific breeding groups that are separated by geography or culture, then these groups may come to have different allele frequencies at a particular genetic locus under study due to differential mutation, selection, or drift. These groups may also have different distributions of the phenotype due to different allele frequencies at other loci (which may or may not be linked to the locus under study), differences in environmental factors, or interaction effects among these factors. In such cases, an association will be observed between genotypes at the locus under study and the phenotype if all groups are analyzed together, even if the locus under study neither causes nor is physically linked to a gene that causes variation in the phenotype (Ewens and Spielman, 1995). This is one of the great Achilles' heels of association studies and motivated the development of the transmission disequilibrium test (TDT) to be discussed below.

\section{Why the Recent Interest in Association Studies?}

If association studies have this major Achilles' heel that linkage studies do not, why are they so popular? Some of the reasons for the popularity of association studies are simple and obvious. Association studies are relatively easy to conduct, requiring only unrelated individuals. Moreover, the recent introduction of single nucleotide polymorphisms potentially allows one to conduct a genome-wide association scan with diallelic marker loci (Chakravarti, 1998). Third, a number of

\section{An Illustration of Simpson's Paradox}

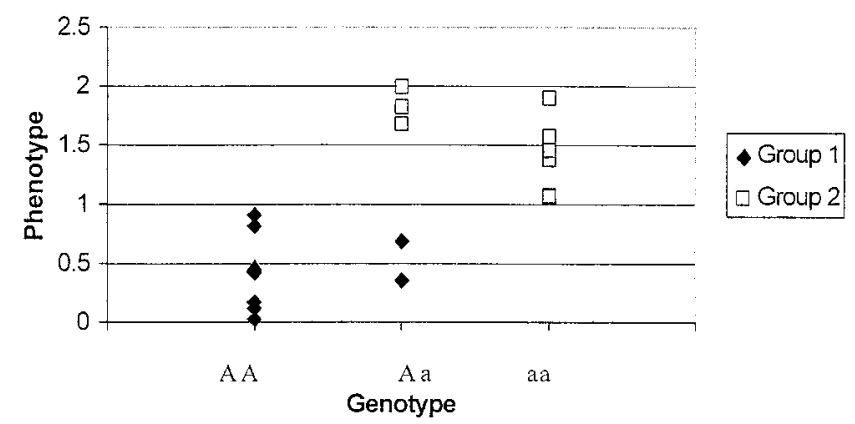

FIG. 1. An illustration of Simpson's paradox. There is no association between genotype and phenotype within groups, but when both groups are combined, a spurious (due to confounding) association is observed. investigators have sampled data from admixed populations and population isolates, both of which may show more linkage disequilibrium than other populations and may therefore be better suited to association mapping (Briscoe et al., 1994; Hastbacka et al., 1992). Fourth, as mouse genetics has progressed in certain areas, such as obesity (Robinson et al., 2000), ever larger and more credible lists of candidate genes have become available for investigation in association studies. Fifth, now that several credible linkages have been found for a number of quantitative traits in humans (e.g., obesity; Perusse et al., 2001), there is a desire to narrow those regions through fine mapping based on disequilibrium (Kaplan and Morris, 2001). Finally, under certain circumstances, association tests can be enormously more powerful than linkage studies. This will almost certainly be true in circumstances where one has a candidate gene either for which there is a candidate polymorphism or which is diallelic and may be true in other circumstances as well. To see why this is so, consider the following unrealistic example simplified for expository purposes.

Imagine a trait that is influenced by only one factor, a single diallelic quantitative trait locus (QTL) with alleles $A$ and $a$ having frequencies $(1-p)$ and $p$, respectively. Imagine further that the QTL acts in a fully additive fashion and, without loss of generality, that the mean of the $A A$ genotype is zero. Denoting the mean of the $a a$ genotype as $\mu_{a a}$, the mean of the $A a$ genotype is then $1 / 2 \mu_{a a}$. In this situation, the correlation between genotype and phenotype will be 1.0 and, therefore, the power is perfect. In contrast, consider what would occur if one conducted a sibling-pair linkage analysis in which one assessed whether some function of sibling-pair phenotypic similarity was associated with the number of alleles the pair shared IBD. Even under the ideal circumstances of a perfectly informative marker that is completely linked to the QTL, there will be an imperfect relationship between the number of alleles a pair shares IBD and the number of alleles the pair shares identical in state IIS. ${ }^{2}$ The relation is given in Table I.

Table I points out that even under the unrealistic and idealized situation in which there is a perfect linear relation between phenotype and genotype, a test of linkage will not have perfect power. This is because, within the IBD classes of zero and 1, there will be variability in the IIS status of sib pairs and it is IIS status that determines the expected value of phenotypic similarity (or dissimilarity). This in turn implies that there will be variability

\footnotetext{
${ }^{2}$ IIS is more commonly referred to as IBS (identical by state) in the literature.
} 


\section{TABLE I}

Probability of IIS Status Given IBD Status in Sibling Pairs and Squared Intrapair Phenotypic Difference Given ISS ${ }^{a}$

\begin{tabular}{|c|c|c|c|c|c|}
\hline & $\mathrm{IBD}=0$ & $\mathrm{IBD}=1$ & $\mathrm{IBD}=2$ & $E\left(X_{1}-X_{2}\right)^{2} \mid$ IIS & $\operatorname{Var}\left(X_{1}-X_{2}\right)^{2} \mid$ IIS \\
\hline$P(\mathrm{IIS}=0 \mid \mathrm{IBD})$ & $2(1-p)^{2} p^{2}$ & 0 & 0 & $\mu_{a a}^{2}$ & 0 \\
\hline$P(\mathrm{IIS}=1 \mid \mathrm{IBD})$ & $4\left[(1-p)^{3} p+p^{3}(1-p)\right]$ & $2 p(1-p)$ & 0 & $\left(\frac{1}{2} \mu_{a a}\right)^{2}$ & 0 \\
\hline$P(\mathrm{IIS}=2 \mid \mathrm{IBD})$ & $(1-p)^{4}+(2 p(1-p))^{2}+p^{4}$ & $1-2 p(1-p)$ & 1 & 0 & 0 \\
\hline$E\left(X_{1}-X_{2}\right)^{2} \mid \mathrm{IBD}$ & $\begin{array}{l}2(1-p)^{2} p^{2} \mu_{a a}^{2} \\
\quad+4\left[(1-p)^{3} p+p^{3}(1-p)\right]\left(\frac{1}{2} \mu_{a a}\right)^{2}\end{array}$ & $2 p(1-p)\left(\frac{1}{2} \mu_{a a}\right)^{2}$ & 0 & & \\
\hline $\operatorname{Var}\left(X_{1}-X_{2}\right)^{2} \mid \mathrm{IBD}$ & $\begin{array}{rl}\sum_{k=0}^{2} P & P(\mathrm{IIS}=k \mid \mathrm{IBD}=0) \\
& \times\left[\left(E\left(X_{1}-X_{2}\right)^{2} \mid \mathrm{IIS}=k\right)\right. \\
& \left.-\left(E\left(X_{1}-X_{2}\right)^{2} \mid \mathrm{IBD}=0\right)\right]^{2}\end{array}$ & $\begin{array}{rl}\sum_{k=0}^{2} P & P(\mathrm{IIS}=k \mid \mathrm{IBD}=0) \\
& \times\left[\left(E\left(X_{1}-X_{2}\right)^{2} \mid \mathrm{IIS}=k\right)\right. \\
& \left.-\left(E\left(X_{1}-X_{2}\right)^{2} \mid \mathrm{IBD}=0\right)\right]^{2}\end{array}$ & 0 & & \\
\hline
\end{tabular}

${ }^{a}$ The calculations of the expected value and variance of $\left(X_{1}-X_{2}\right)^{2}$ given IIS or IBD status assume a heritability of 1.0 at the trait locus.

in some nonzero residual variance from the regression of functions of phenotypic similarity on IBD status. Thus, a perfect correlation in an association analysis becomes a less than perfect correlation, with obviously less than perfect power, in a linkage analysis. ${ }^{3}$ This decrement in power will also be present when the QTL does not explain $100 \%$ of the phenotypic variance, but is easiest to see in this hypothetical case.

\section{The Original Transmission Disequilibrium Test}

Given the marked advantages of association studies over linkage studies (under some conditions), association studies would be extraordinarily valuable if one could eliminate the potential confounding effects of population stratification. This is just what the original TDT did for dichotomous traits such as diseases (Spielman et al., 1993). The key development in Spielman et al.'s TDT was more design than test. The test is none other than the well-established McNemar (1947) test. The great insight that Spielman et al. had was that by applying McNemar's test to test whether a putative disease allele was transmitted to an offspring from a heterozygous parent, one had essentially set up a situation in which transmission of a portion of a chromosome containing a particular allele from parent to child became a random event with equal

\footnotetext{
${ }^{3}$ It is also evident that the linkage context, in the case of sib pairs, reduces the number of units of observation by one-half, which on a person-per-person basis reduces power further. Moreover, regressing intrapair similarity on IBD status introduces heteroscedasticity (because the variance of intrapair similarity is not constant for all IBD classes) which may also affect power adversely.
}

probability, which we will denote $P(t)$, for all offspring in the sample. That is, by only counting transmissions from heterozygous parents, one has effectively conditioned on parental genotype and made the probability of being transmitted a disease allele equal for all offspring. In essence, the act of gametogenesis becomes the random assignment mechanism in which offspring are randomly assigned to receive or not receive a portion of a chromosome containing a putative disease allele and this act of random assignment ensures that one can make valid causal inferences (Rubin, 1991). That is, if there is an association between being transmitted a portion of a chromosome containing a putative disease allele and having a disease, then there must be a causal relation between either the putative disease allele or something physically linked to it on the chromosome and development of the disease phenotype. Thus, by conducting association analyses conditional upon parental genotype we can conclude that a putative disease allele is both linked to and associated with a phenotypic characteristic. To complete the development of the original TDT, Spielman et al. (1993) built on one additional assumption, namely that the probability of inheriting either of the two alleles at homologous chromosomes from a heterozygous parent was equal (i.e., 1/2) for each allele. This is the assumption of no segregation distortion. In the event that one is not prepared to accept the assumption of no segregation distortion, and there is evidence that segregation distortion does occur (Yasue et al., 1999), Spielman et al. (1993) point out that one can include unaffected offspring in the analysis to account for this. However, if we accept that there is no segregation distortion, then we are stating that $P(t)$ is $1 / 2$ in the total 
population. Furthermore, if there is an association between inheritance of a putative disease allele and a dichotomous phenotype, then, by definition, $P(t)$ is not equal for subsets of the population with different values of the dichotomous phenotypes (e.g., diseased vs not diseased people). Finally, if, in two subsets of the population, $P(t)$ is not equal, but $P(t)$ does equal $1 / 2$ in the whole population, then $P(t)$ cannot equal $1 / 2$ in the subsets. Thus, simply observing that $P(t)<>1 / 2$ in a subset of the population defined by phenotypic status is tantamount to directly observing an association between transmission and phenotypic status if and only if one assumes no segregation distortion. Thus, it can be seen that joint tests of linkage and association ${ }^{4}$ can be grouped into two categories. One category directly examines the association between transmissions from a heterozygous parent to offspring phenotype (which is just a special case of observing an association between offspring's genotype and offspring's phenotype conditional on parental genotypes). The other category only infers the existence of such association by observing a deviation from the transmission probability of $1 / 2$ and assuming no segregation distortion. It should be possible to combine both types of tests into a more powerful weighted composite when, for example, offspring have been selected for having only moderately elevated (or depressed) scores on the quantitative trait under study. A study has been done for allele sharing linkage statistics (Allison et al., submitted; Forrest and Feingold, 2000), but the details of such combined tests have yet to be formally presented.

\section{THE FIRST TDTS FOR QUANTITATIVE TRAITS}

As stated above, the original TDT eliminates potential confounding due to population stratification by effectively conditioning on parental genotypes. Building on this realization, Allison (1997) extended the TDT to quantitative traits. Allison developed five TDTs for quantita-

\footnotetext{
${ }^{4}$ Herein we will use the phrases "joint test of linkage and association," "test of linkage in the presence of association," and "test of association in the presence of linkage" interchangeably to mean a test which will yield $P$ values less than some level $\alpha$ greater than $100 \alpha \%$ of the time if and only if the marker locus under study is both linked to a locus that produces variation in the phenotype under study and allelic variation at the marker locus is associated with allelic variation at the phenotype-influencing locus. Any test properly called a TDT is such a test. However, not all legitimate joint tests of linkage and association are referred to as TDTs.
}

tive traits dubbed TDTQ1 to TDTQ5. Several of those TDTs were presented by Allison (1997) primarily for pedagogical purposes as they lead to the development of TDTQ5, which is generally the most flexible and powerful of the five. Rabinowitz (1997) independently developed a TDT for quantitative traits that has certain advantages in being nonparametric and easily adaptable to multiple offspring and multiple alleles. Herein, we will discuss only TDTQ4 and TDTQ5.

TDTQ4 is nothing more than the trivial extension of the original TDT to a quantitative trait made dichotomous. For example, if we were studying obesity, we might have a sample of subjects who were all selected for having body mass indices (in kilograms per square meter) above 30 . We could then call all of these people "affected" with obesity and simply conduct the original TDT.

TDTQ5 is only slightly more complex. In TDTQ5, the quantitative phenotype is kept continuous and is regressed on offspring genotypes while controlling for parental mating types. Parental mating types are defined on the basis of parental genotypes. Because only families with at least one heterozygous parent are informative in this type of TDT analysts (Ewens and Spielman, 1995), with a diallelic locus, there are three relevant mating types defined by the parental genotypes $A a, A a ; A A, A a$; and $A a, a a$. One codes the three parental mating types into two dummy variables and enters these two dummy variables into a regression analysis. Then, one dummy codes the three possible offspring genotypes ( $A a ; A A$; and $a a$ ) into two dummy additional dummy variables and enters them into the regression equation. The 2-df $F$ test for the joint effect of these latter two dummy variables can be used to assess the significance of the evidence that the marker locus being evaluated is both linked to and associated with the phenotype. Thus, TDTQ5 is nothing more than ordinary multiple regression. Recognizing this has two very desirable results. First, it means that one can use commonly available, welldocumented, and well-tested software such as SAS, SPSS, or S-PLUS to conduct one's analysis. Second, it means that the very large fund of knowledge and "bag of tricks" that exist in the literature regarding regression analysis can be used (c.f., Neter et al., 1990). This includes incorporation of covariates, testing of interactions, running robust regressions, etc.

A few notes about TDTQ5 are in order. Many TDTs and other association tests are formulated to only detect the allelic or additive effects (see Lynch and Walsh, 1997, for definitions of these terms). In situations in which all or most of a QTL's variance is additive, such approaches will generally yield more power than the approach 
described above for TDTQ5 which assesses the genotypic effects. This is because assessing the genotypic effects requires $2 \mathrm{df}$ whereas assessing the allelic effects only requires $1 \mathrm{df}$. We consider using the 2-df test appropriate for two reasons. First, in the event that a large proportion of the QTL variance is nonadditive (i.e., dominance variance), the regression modeling genotypic effects will better fit the data and therefore, the 2-df test of genotypic effects will be more powerful. Second, it is our expectation that most investigators will, if the 1-df test for additive effects is not significant, try the 2-df test which creates a multiple testing problem if not taken into account. Therefore, we believe it is more "honest" to simply conduct the 2-df test up front. Nevertheless, if one wanted to test only for additive effects, this can easily be done by simply coding the offspring genotypes as a single variable with values 0,1 , and 2 denoting the number of $a$ alleles the offspring has instead of dummy coding two variables for genotype.

\section{Extensions and Variations}

Multiple alleles. TDTs are often applied to candidate genes or SNPs where the loci being tested are diallelic. However, in some cases one may wish to conduct TDTs with multiallelic loci. Most TDTs are easily extended to accommodate multiallelic loci (Allison et al., 1997; Horvath et al., 2001; Laird et al., 2000; Rabinowitz et al., 1997). In the case of TDTQ5, this can be accomplished by simply creating $k-1$ dummy codes where $k$ is the number of different offspring genotypes observed at the locus under study. Similarly, one must dummy code $M-1$ variables where $M$ is the number of different parental mating types observed at the marker locus. Although conceptually simple, if the number of different alleles is large, the loss in denominator df, the increase in numerator $\mathrm{df}$, and the small cell size for many genotypes may result in unacceptably low power. In such situations, methods that seek the allele with greatest effect in a multiallele locus while controlling for multiple testing (Betensky and Rabinowitz, 2000; Kaplan et al., 1997) may be adapted.

Multiple loci. There is increasing recognition of the need for and benefits of modeling the joint effects, both additive and epistatic, of multiple loci (Frankel and Schork, 1996; Goldgar and Easton, 1997; Marinov and Weeks, 2001). In most TDTs for quantitative traits, multiple loci can be easily accommodated. In the case of TDTQ5, this simply entails expanding the regression to test for the effects of dummy coded offspring genotypes at each locus of interest after controlling for additional dummy coded parental mating types at each locus. Epistatic effects can be tested by coding product terms among the loci for which one wishes to test interactions (Aiken and West, 1991).

Logistic regression. One variation on TDTs for quantitative traits comes from Waldman et al. (1999) and involves using logistic regression instead of ordinary linear regression. In this approach, the dependent variables and independent variables are essentially reversed and the unit of analysis becomes the heterozygous parent. In the case of a diallelic locus, it would involve coding as 1 those cases in which, for example, $a$ was transmitted and as 0 those cases in which $A$ is transmitted. In the event of a family with two heterozygous parents, both are treated as separate cases, which is legitimate because, under the null hypothesis or when subjects are randomly sampled, which allele is transmitted by one heterozygous parent is independent of which allele is transmitted by the other heterozygous parent (Spielman et al., 1993). The offspring's phenotype is then used as the independent variable. This approach has several advantages. First, like TDTQ5, this is easily implemented in standard statistical software. Second, no distributional assumptions need to be made about the phenotype for validity of the test, i.e., it is nonparametric. Third, it is easily extended to multivariate phenotypes by simply including additional phenotypes as predictors in the regression. Fourth, it easily accommodates multiple offspring per nuclear family. The primary disadvantages of the method are that (1) it should be less powerful than parametric tests such as TDTQ5 when all parametric assumptions are met, (2) it does not easily accommodate multiple loci or multiallelic loci (although it could be made to do so through the use of multiple-group logistic regression (Cohen and Rom, 1994), and (3) it tests only for allelic effects, not genotypic effects.

Multiple phenotypes. Many investigators collect information on multiple phenotypes, but most only analyze one phenotype at a time. This is unfortunate for two reasons. First, the potential power advantages of multivariate analyses are well documented (see Allison et al., 1998, and references therein). Second, if many phenotypes are all analyzed separately, then the experiment-wise type 1 error rate may increase to an unacceptable rate if correction is not applied (Allison and Beasley, 1998). Thus, it is desirable to conduct multivariate analyses when possible. In the case of Waldman et al.'s (1999) logistic regression approach, as described above, this is easily accomplished by including multiple phenotypes as predictor variables. In the case of TDTQ5, 
this is also easily accomplished by switching to multivariate analysis of variance (MANOVA), of which multiple regression is just a special case (Woodward et al., 1990). For quantitative TDTs where the generalization to multivariate analysis is not immediately obvious, adaptation of an approach due to Mangin et al. (1998) can always be used. In brief, this approach entails subjecting the $c$ phenotypes to a principle components analysis and extracting $c$ orthogonal components. Each of the $c$ components can then be analyzed as a dependent variable in a univariate analysis. The $c$ resulting tests statistics will be independent and can then, if expressed as $\chi^{2}$ s, be summed to produce an omnibus test statistic that will also be distributed as $\chi^{2}$ with df equal to the summation of the df across each of the $c$ individual $\chi^{2}$ 's.

Parent of origin/imprinting effects. For some phenotypes, like body composition and obesity traits (Dhar et al., 2000; Georges and Cockett, 1996; GunayAygun et al., 1997), there is reason to suspect that there may be imprinting effects. In the case of TDTQ5, this can be tested for by coding an additional dummy variable to indicate whether the father is heterozygous or the mother is heterozygous in parent couples where only one is heterozygous and then testing for interactions between this variable and offspring genotype. Most other methods can also be adapted to evaluate imprinting effects (e.g., van den Oord, 2000; Zhao, 2000).

Multiple offspring. In many data sets, investigators have multiple offspring within nuclear families. In such cases, it is wise to use all available data. Waldman et al.'s logistic regression approach can easily accommodate multiple offspring as described above. In analytic approaches in which the phenotype(s) remains the dependent variable, utilizing multiple offspring per nuclear family is also possible, but slightly more challenging. Including each offspring in the analysis as though it was independent would be inappropriate because residuals are likely to be correlated within families (Lake et al., 2000) and ignoring this would violate a critical assumption (Berry, 1993). The potential correlation among siblings' residuals can be accommodated in a test like TDTQ5 by switching from ordinary least squares regression to iteratively reweighted generalized least squares regression (Sen and Srivastava, 1990) or any similar method (e.g., Lake et al., 2000) that estimates and allows for the residual correlation among siblings such as through a mixed model (e.g., Goddard, 1992) or GEE (Bull et al., 1995). It is important to point out that simply conducting an analysis through a method such as GEE or a mixed model that accounts for the correlation among siblings does not automatically make what one is doing a "TDT" or a legitimate test of linkage in the presence of association. One must still, as Rabinowitz and Laird (2000) put it, compare "test statistics for association to their conditional distributions given the minimal sufficient statistic under the null hypothesis for the genetic model, sampling plan and population admixture." Multiple siblings can also be accommodated through combined maximum likelihood variance components association and linkage analysis (e.g., Neale et al., 1999) or via maximum likelihood through the SAGE ASSOC software as described by Zhu and Elston (2000) and below.

Large pedigrees. There may also be situations in which investigators have pedigrees that go beyond nuclear families and wish to apply a TDT for quantitative traits to the entire data set. In this situation, the parent in one unit suitable for TDT analysis may be the offspring in another unit, or offspring from different units might be, for example, cousins. The challenge posed in this situation is just a more complex version of the one posed by having multiple siblings. The issue, as with siblings, is that all residuals cannot be assumed to be independent. However, with larger pedigrees, the situation is more challenging. With siblings, all related individuals whose phenotypic measurements are treated as dependent variables have the same relation (i.e., siblings) and can therefore be expected to have a common residual correlation. However, in complex pedigrees there will be many different relations among individuals whose phenotypic values are treated as dependent variables in the analysis. Although this can, in theory, be taken into account using all of the general approaches mentioned above for siblings, in practice, estimating the now complex covariance matrix among the residuals is difficult and the task is often best approached by using specialized software designed for large pedigrees as illustrated elsewhere (e.g., George et al., 1999a, b; Zhu et al., 2001; Zhu and Elston, 2000).

When one parent's genotypic information is missing. Curtis and Sham (1995) were the first to point out the need for specialized approaches when genotypic information is not available for one parent in the context of the original TDT. Similarly, in quantitative TDTs there is a need to adapt to situations where one parent's genotype is missing. If one were willing to assume that parents' genotypes are missing completely at random or missing at random (in the terminology of Little and Rubin, 1987), then the whole host of legitimate missing data techniques, including use of the EM algorithm and multiple imputation that have been developed in the broader statistical literature (see Little and Rubin, 1987; Schafer, 1997), can be brought to bear. Thus, for 
example, TDTQ5 could be applied to data set in which some of the parents' genotypes were missing by augmenting the data set through imputation multiple times, then analyzing each "pseudo-complete" dataset in the standard way, and then combining the results, adjusting for the imputation as described by Schafer (1997) and illustrated by $\mathrm{Heo}$ et al. (in press). Moreover, just as TDTs for dichotomous traits were developed that specifically took missing parental information into account (e.g., Knapp, 1999; Weinberg, 1999), now most TDTtype tests (or more generally family-based tests of linkage in the presence of association) developed for quantitative traits are routinely designed to allow for missing parental genotypes (e.g., Horvath et al., 2001; Rabinowitz and Laird, 2000; van den Oord, 2000; Whittemore and Tu, 2000).

\section{Sibling-Based TDTs for Quantitative Traits}

After the development of the original TDT, several investigators realized that they could construct legitimate tests of linkage in the presence of association for dichotomous traits even when no parental data were available by relying on sibling data (Boehnke and Langefeld, 1998; Curtis, 1997; Curtis et al., 1999; Ewens and Spielman, 1998; Horvath and Laird, 1998; Horvath et al., 2000; Schaid and Li, 1997; Schaid and Rowland, 1998). This is an important development because many traits (e.g., sarcopenia; Morley et al., 2001) are only manifest or commonly studied among elderly persons whose parents are unlikely to be available for genotyping.

Because full siblings, by definition, share the same parents, controlling for the sibship an individual is from automatically controls for their parents' genotypes. Thus, if association studies in which there are multiple siblings per sibship are conducted conditional upon sibship, then these will yield legitimate tests of linkage in the presence of association. Technically, if there are more than two siblings per sibship, such tests remain legitimate tests of linkage that capitalize on any association present but may not remain legitimate tests of linkage (Ewens and Spielman, 1998). In practice, most investigators seem unconcerned about this "technicality," apparently because they would be happy to find linkage even in the absence of association.

Once one realizes that association studies conducted conditional upon sibship are legitimate TDTs, then it is easy to design sibling-based TDTs for quantitative traits (or for that matter any trait such as "time to event" (e.g., Mokliatchouk et al., 2000). All that is needed is to conduct an analysis in which association between the marker locus and the quantitative trait is assessed after controlling for sibship. Allison et al. (1999) offered two such tests. One is simply a mixed-model ANOVA in which genotype is the fixed factor and sibship is the random factor. This has all the same advantages as TDTQ5 has, including the fact that it is extremely flexible (e.g., allows multiple alleles, multiple loci, interaction effects, covariates, etc.) and can be implemented in standard software (e.g., SAS, SPSS, S-PLUS). The second procedure they offered is a permutation test that has the advantage of making no distributional assumptions regarding the phenotype or its residual. Schaid and Rowland (1999) offered another TDT for quantitative traits that does not require parental information. More recently, a number of "generalized" TDTs (Will the real generalized TDT please stand up?) have been developed, all of which allow inclusion of families with no parental information (Abecasis et al., 2000; Laird et al., 2000; Rabinowitz and Laird, 2000; Schaid and Rowland, 2000; Sun et al., 2000; Zhao and Sun, 1999).

\section{Partitioning the Linkage and Association Information in a Variance Components Framework}

The idea of partitioning variance into components associated with (i) the putative QTL, (ii) other familial factors, and (iii) nonfamilial factors goes back at least to the original Haseman-Elston (1971) regression method. However, in this early treatment the focus was entirely on the QTL component and the residual was of only minor interest. Amos (1992) proposed a variance components model that more formally extracted this information, and Fulker and Cherny (1996) described the model explicitly in terms of the predicted variance-covariance matrix of pairs of siblings. This model specifies the values of the expected $2 \times 2$ phenotypic covariance matrix for groups of sibling pairs as a function of three parameters or variance components. Specifically, the expected variances are equal to

$$
\sigma_{\mathrm{q}}^{2}+\sigma_{\mathrm{r}}^{2}+\sigma_{\mathrm{e}}^{2}
$$

and the expected covariances are equal to

$$
\hat{\pi} \sigma_{\mathrm{q}}^{2}+\sigma_{\mathrm{r}}^{2}
$$

where $\hat{\pi}$ is an estimate of the IBD for the sib pair at the locus, which is computed as $\hat{\pi}=p(\operatorname{IBD}=2)+$ $0.5 p(\mathrm{IBD}=1) ; \sigma_{\mathrm{q}}^{2}$ is variance due to the QTL; $\sigma_{\mathrm{r}}^{2}$ is residual variance, both genetic and environmental, shared by members of a sib pair; and $\sigma_{\mathrm{e}}^{2}$ is residual variance, including error of measurement, not shared by siblings. 
The IBD probabilities are based on the genetic marker data and may be computed by software such as Genehunter (Kruglyak and Lander, 1996) or Merlin (Abecasis and Cardon, unpublished). On average, $\hat{\pi}$ will equal 0.5 in randomly selected samples of sib pairs. If the putative QTL causes variance in the trait, or is linked (with recombination fraction $<0.5$ ) to a locus that does, there is a predicted difference in sib pair resemblance, such that those with higher values of $\hat{\pi}$ will have a higher predicted

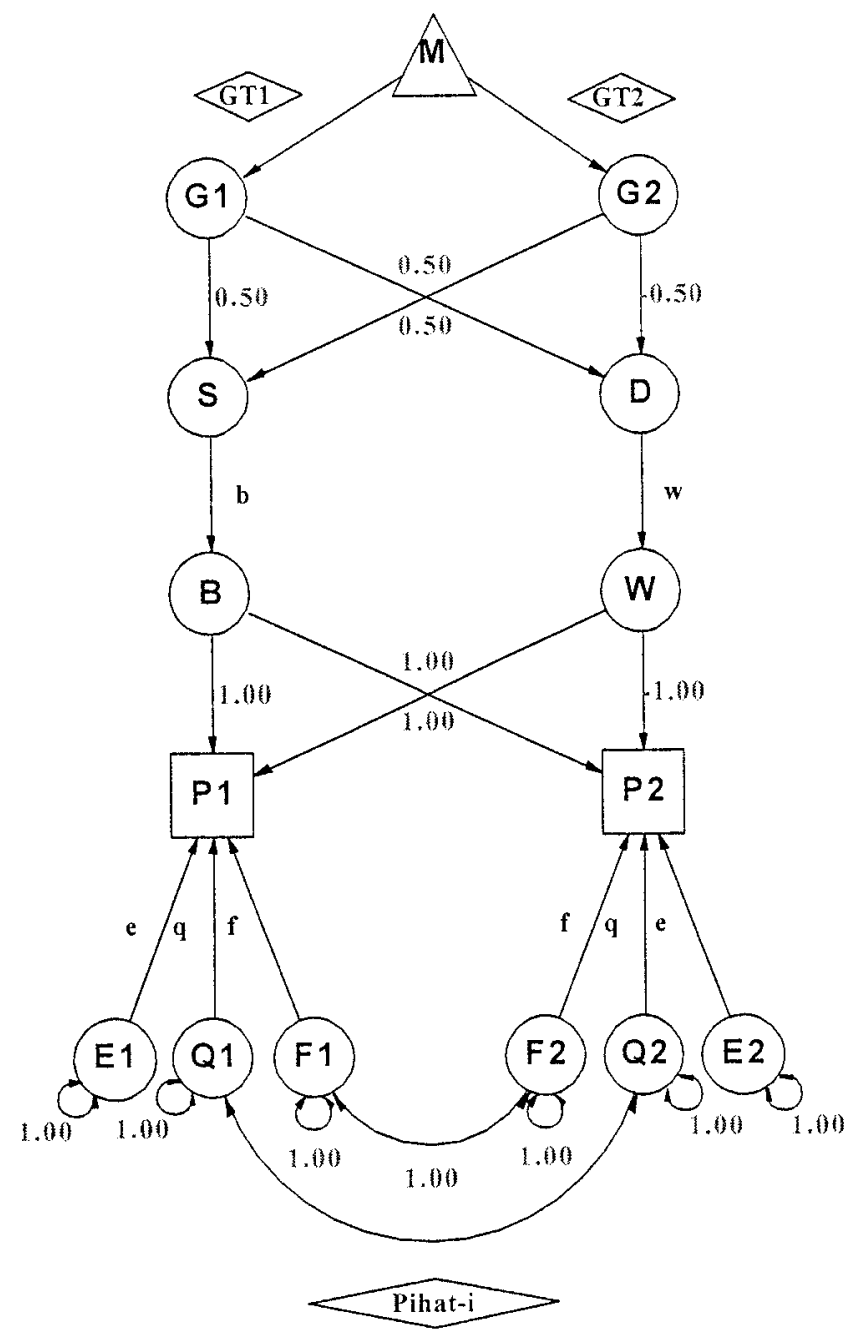

FIG. 2. Path diagram of model for joint linkage and association in sib pairs, allowing for possible population stratification. Latent variables for family resemblance $F$, QTL variance $Q$, and individualspecific variance $E$ cause the phenotypes of two siblings, P1 and P2. $S$ represents half the sum of the sibling pair's genotypic effects, and $D$ represents half their difference. These components contribute to between- (B) and within-pair effects (W) via parameters $b$ and $w$, respectively. In the absence of stratification, $b$ and $w$ are expected to be equal. Genuine association with observed genotypes GT1 and GT2 will decrease the size of the linkage-based QTL effect, $q$. covariance. An interesting aspect of this model is that it is a type of structural equation model with a moderated covariance parameter. Figure 2 shows a path diagram of the model. Note how the covariance between the latent variables Q1 (the effect of QTL on sib 1 phenotype P1) and Q2 is set to be the observed value of $\hat{\pi}$ for this location on the genome. That is, each sib pair in the sample may have a different predicted covariance matrix. Moderated structural equation models of this type were proposed by Neale and Cardon (1992) and implemented in $\mathrm{Mx}$ (Neale, 1994) shortly afterward.

An alternative approach to the $\hat{\pi}$-based specification of the effects of linkage was described by Fulker and Cherny (1996). Rather than summarizing the IBD probabilities into a single $\hat{\pi}$ statistic, these probabilities are used to weight the likelihood under each model. That is, the model specifies that sib pairs come in three types and share exactly zero, one or two alleles IBD, not any value in between. The likelihood of a pair of scores is then calculated conditional on these states. That is, there are three predicted covariances between siblings: $r^{2}+q^{2}$ for the IBD $=2$ case, $r^{2}+0.5 q^{2}$ for the IBD $=1$ case, and $r^{2}$ for the IBD $=0$ case. The likelihood is computed as the weighted sum of the likelihoods under these three models, where the weights are the IBD probabilities. This alternative formulation appears to have greater statistical power, particularly when the QTL effect is large, and it also appears to be more robust to the effects of selected sampling (Dolan et al., 1999). However, it is more difficult to generalize this approach to studies of larger pedigrees, because the number of IBD configurations increases from three in the case of sib pairs to $10,36,136$, and 528 for sibships of sizes $3,4,5$, and 6 , respectively. In general, the number of IBD configurations - which is the number of components in the mixture distributionincreases according to the function $2^{2 m-3}+2^{m-1}-2^{m-2}$ for sibships of size $m$. This exponential series causes some practical and computational difficulties for the implementation of the mixture distribution approach in large pedigrees.

Association in the QTL structural equation model. A more recent innovation in the analysis of quantitative traits is the incorporation of possible association with a specific marker locus. In its most trivial form, this model simply involves the specification of a different mean for each genotype. Usually, structural equation models do not make explicit predictions about means. However, since Sorbom (1974) showed that means could be included, and because certain structural equation models, particularly those dealing with development over time (Dolan et al., 1989; Neale and McArdle, 2000), make predictions 
about means, this extension has become more common. Of special note is that the more recent advent of fitting structural equation models to raw data by maximum likelihood (Neale, 1994; Arbuckle, 1997) requires specification of a model for the means. Often, models for the means are saturated, in that every mean is allowed to take its own value. In variance components models, this saturated model is usually restricted such that variables of the same type, such as all male siblings measured on the same variable, are equated. It is a straightforward extension of this model that permits a different mean for individuals of different genotypes. Furthermore, this modeling of means can be added to the model for linkage, as shown in Fig. 2.

As noted above, a simple association model of different means for different genotypes is subject to the confounding effects of population stratification. Fulker and Cherny (1998) (see also Neale et al., 1999; Neale, 2001) showed how the stratification can be removed by fitting models that specify separate mean components between families and within families. The general idea is that population stratification will cause differences between families but not within them, because individuals from the same family are assumed to originate from the same stratum of the population. Allelic effects, by contrast, will occur regardless of whether a difference in alleles occurs within or between families. That is, the betweenfamily effect will be equal to the within-family effect if there is no population stratification. To model this situation, two allelic effects are specified per locus. First, $b$ is defined as the mean genotypic effect of the pair. For example, if both members of the pair have genotypes with deviations $+b$, their mean will be $b$. These effects are shown in column 5 of Table II. Second, allelic effects within pairs are defined as half the difference (Sib1-
Sib2)/2 between their genotypic deviations, and these deviations are shown in column 6 of Table II. Noting that pair mean is $(\mathrm{Sib} 1+\mathrm{Sib} 2) / 2$, we can deduce that the expected mean of Sib1 is the mean plus half the difference, and the expected mean of Sib2 is the mean minus half the difference. These quantities are shown in columns 6 and 7 of Table II. An interesting consequence of modeling the means is that when there is an effect on the mean, the amount of variance to be explained is reduced. For example, consider two groups, with normal distribution and equal variance, but with means that differ by 4 standard deviations. The variance of the two groups together is much larger than the variance within each group. Once the groups' means have been modeled by allowing a mean effect associated with group membership, the residual variance is greatly attenuated.

At this point it is evident that joint modeling of the means, attributed to the putative QTL, and of the covariances as a function of the IBD sharing probabilities, is possible. Cardon and Abecasis (2000) illustrate the method with simulated data and show how the evidence for linkage decreases when the mean effect of the QTL is modeled. In addition, this decrease occurs only in a region in tight disequilibrium with the QTL. Furthermore, the allele frequencies at the locus being tested and at the QTL itself need to be equal to maximize the probability of finding an association signal. Therefore the method is very promising for fine mapping.

Being a linear model, it is possible to represent the model for the means and for the covariances as a structural equation model. Figure 2 shows this model for two sibling phenotypes, P1 and P2, their latent QTL genotypes, Q1 and Q2, residual familial resemblance factors, $\mathrm{F} 1$ and $\mathrm{F} 2$, and specific environment factors, E1 and E2. The covariance between the sibling genotypes, $\hat{\pi}_{i}$, which

\section{TABLE II}

Expected Sib Pair Means and Differences at a Single Additive Two-Allele Locus

\begin{tabular}{|c|c|c|c|c|c|c|c|}
\hline \multicolumn{2}{|c|}{ Genotype } & \multicolumn{2}{|c|}{ Individual Mean } & \multicolumn{2}{|c|}{ Pair Statistics } & \multicolumn{2}{|c|}{ Stratified Means } \\
\hline Sib 1 & Sib 2 & Sib 1 & Sib 2 & Sum $/ 2$ & Difference $/ 2$ & Sib 1 & Sib 2 \\
\hline$A \_1 A \_1$ & $A \_1 A \_1$ & $a$ & $a$ & $a$ & 0 & $b$ & $b$ \\
\hline$A \_1 A \_1$ & $A \_1 A \_2$ & $a$ & 0 & $a / 2$ & $a / 2$ & $b / 2+w / 2$ & $b / 2-w / 2$ \\
\hline$A \_1 A \_1$ & $A \_2 A \_2$ & $a$ & $-a$ & 0 & $a$ & $w$ & $-w$ \\
\hline$A \_1 A \_2$ & $A \_1 A \_1$ & 0 & $a$ & $a / 2$ & $-a / 2$ & $b / 2-w / 2$ & $b / 2+w / 2$ \\
\hline$A \_1 A \_2$ & $A \_1 A \_2$ & 0 & 0 & 0 & 0 & 0 & 0 \\
\hline$A \_1 A \_2$ & $A \_2 A \_2$ & 0 & $-a$ & $-a / 2$ & $a / 2$ & $-b / 2+w / 2$ & $-b / 2-w / 2$ \\
\hline$A \_2 A \_2$ & $A \_1 A \_1$ & $-a$ & $a$ & 0 & $-a$ & $-w$ & $w$ \\
\hline$A \_2 A \_2$ & $A \_1 A \_2$ & $-a$ & 0 & $-a / 2$ & $-a / 2$ & $-b / 2-w / 2$ & $-b / 2+w / 2$ \\
\hline$A \_2 A \_2$ & $A \_2 A \_2$ & $-a$ & $-a$ & $-a$ & 0 & $-b$ & $-b$ \\
\hline
\end{tabular}


varies across sibling pairs, is shown in a diamond because it is an observed variable that is used to define the model. Similarly, measured genotypes GT1 and GT2 are shown in diamonds because they are also measured variables that vary across pairs. The path diagram generates predicted means that agree with the expectations in Table II as long as the diallelic genotypes are scored $-1,0$, and 1 . This model is readily extensible to multiple phenotypes and to larger pedigrees, although it is more efficient to program it using command language than by developing an elaborate path diagram.

Another valuable feature of this maximum likelihood variance components approach is that unbalanced pedigrees that include missing data - even data on single unrelated individuals - may be used in combination with cases that contain complete data. While unrelated individuals do not yield information about linkage, they are informative about association and may rely on the family data to remove the confounding between population stratification and genuine allelic association.

Finally, the variance components approach may be used with binary or ordinal data, when, for example, a threshold model is used to delineate the ordered categories. For many traits of interest to the behavioral scientists, or diagnosis-level data, it is difficult or impossible to obtain measures that are truly based on an interval scale, even if the underlying liability to the trait is normally distributed. Under these circumstances, the application of ordinal data methods may be the only appropriate analysis to conduct. Unfortunately, ordinal level measurement carries the burden that statistical power to detect familial resemblance is drastically reduced (Neale et al., 1995). While potentially crippling for the linkage component of the analysis, there may still be substantial power for the association component.

\section{NON-FAMILY-BASED TESTS OF LINKAGE IN THE PRESENCE OF ASSOCIATION}

The TDT-type approaches described above have one important disadvantage. Specifically, they do not make full use of all available information in the data set. This occurs in many situations, but the most obvious one for illustration is the case of a single individual unrelated to all other individuals in the data set. Such an individual offers no information that can be used in most of the methods for family-based association tests. Such tests, therefore, although very powerful per informative person
(Allison, 1997), are less powerful than ordinary association tests because not all individuals are informative (Long and Langley, 1999).

The "new kids on the block" in terms of joint tests of linkage and association include two broad classes of techniques that attempt to control for potential population stratification without requiring family data (e.g., Bacanu et al., 2000; Devlin and Roeder, 1999; Pritchard and Rosenberg, 1999; Pritchard et al., 1999, 2000a, b; Reich and Goldstein, 2001). This is an extremely important development because, in many contexts, it is difficult if not impossible to obtain family data. Moreover, it may prove to be the case that in some situations the cost of conducting studies using related individuals will exceed the cost of studies yielding equivalent information and power based only on unrelated individuals (Morton and Collins, 1998).

The important advantages of these approaches are that they allow one to conduct legitimate tests of association in the presence of linkage, are not confounded by admixture or stratification, do not require data from related individuals, and utilize all available subjects. A disadvantage of all of the non-family-based tests of linkage in the presence of association is that they require that many markers throughout the genome be typed. Thus, whereas the family-based (TDT-type) approaches require certain types of related individuals, the nonfamily-based approaches require that many loci be typed.

\section{Conditioning on Estimates of Strata or Admixture}

One approach involves estimating an individuals "background admixture" from many loci and then conducting tests of association conditional upon admixture rather than by conditioning on parental genotypes at the specific locus under study as in the TDT approaches. This is the approach taken by Pritchard and colleagues (Pritchard and Rosenberg, 1999; Pritchard et al., 1999, 2000a, b) and Satten et al. (2001). As we see it, the principle behind this approach is conceptually very closely related to Rosenbaum and Rubin's (1984) propensity score approach. The approach by Satten et al. is especially creative and starts from the assumption that, if there were no population substructure or stratification, then there would be no opportunity for confounding and there would also be no disequilibrium among unlinked loci. Given this assumption, if one found a population with no disequilibrium among unlinked loci and, within that population, found a pair of loci that were in disequilibrium, then the two loci in question must be linked. Satten et al. then use a version of latent class analysis to define classes of people in a sample among whom there is 
no apparent disequilibrium among unlinked loci. Having established these latent classes, the test of association between a marker of interest and the phenotype is then conducted conditional upon estimated latent class. In actuality, Satten et al. do the entire analysis in a single step. They present extensive simulations in support of the validity of their method. Although this method is exciting and the simulations are impressive, it is important to point out that the method is still based on controlling for an estimated strata and there is always the possibility that, if that estimate is poor, residual confounding (Becher, 1992) will occur. It is likely that the near future will bring much more research to bear on the utility and performance of this technique.

\section{Genomic Control Methods}

The second approach has been called genomic control. This approach involves examining the association statistics at many loci throughout the genome, deriving a form of correction factor from these many associations, and then correcting the statistic at the locus of interest by this factor (e.g., Bacanu et al., 2000; Devlin and Roeder, 1999; Reich and Goldstein, 2001). There are several variations on the class of approach but all have in common that, rather than simply penalizing investigators for conducting many associations as a Bonferonni correction might do, these methods take advantage of the fact that a whole suite of tests have been conducted. To our knowledge, to date, these methods have only been presented for the case of dichotomous phenotypes, but the extension to quantitative phenotypes is quite simple and just involves redefining the pointwise test statistic to be one that measures evidence for association with a quantitative trait. Of course, one can always dichotomize a quantitative trait, but there is no real need to do so and such action typically reduces power (Cohen, 1983).

\section{REFERENCES}

Abecasis, G. R., Cookson, W. O. C., and Cardon, L. R. 2000. Pedigree tests of transmission disequilibrium, Eur. J. Hum. Genet. 8, 545-551.

Aiken, L. S., and West, S. G. 1991. "Multiple Regression: Testing and Interpreting Interactions," Sage, Newbury Park.

Allison, D. B. 1997. Transmission disequilibrium tests for quantitative traits, Am. J. Hum. Genet. 60, 676-690.

Allison, D. B., and Beasley, M. 1998. A method and computer program for controlling the family-wise alpha rate in gene association studies involving multiple phenotypes, Genet. Epidemiol. 15, 87-101.

Allison, D. B., Thiel, B., St. Jean, P., Elston, R. C., Infante, M., and Schork, N. J. 1998. Multiple phenotype modeling in gene mapping studies of quantitative traits: Power advantages, Am. J. Hum. Genet. 63, 1190-1201.

Allison, D. B., Heo, M., Kaplan, N., and Martin, E. R. 1999. Development of sibling-based tests of linkage in the presence of association for quantitative traits that do not require parental information, Am. J. Hum. Genet. 64, 1754-1764.

Allison, D. B., Fernandez, J., Shette, S., and Amos, C. I. 2001. A class of combined covariance-based and marginal-based allelesharing linkage tests for quantitative traits, submitted.

Arbuckle, J. 1997. Amos 3.6 Users' Guide, SmallWaters Corp., Chicago.

Bacanu, S. A., Devlin, B., and Roeder, K. 2000. The power of genomic control, Am. J. Hum. Genet. 66(6), 1933-1944.

Becher, H. 1992. The concept of residual confounding in regression models and some applications, Stat. Med. 11, 1747-1758.

Berry, W. D. 1993. "Understanding Regression Assumptions," Sage, Newbury Park.

Betensky, R. A., and Rabinowitz, D. 2000. Simple approximations for the maximal transmission/disequilibrium test with a multi-allelic marker, Ann. Hum. Genet. 64, 567-574.

Boehnke, M., and Langefeld, C. D. 1998. Genetic association mapping based on discordant sib pairs: The discordant-alleles test, Am. J. Hum. Genet. 62, 950-961.

Briscoe, D., Stephens, J. C., and O’Brien, S. J. 1994. Linkage disequilibrium in admixed populations: applications in gene mapping, J. Hered. 85, 59-63.

Bull, S. B., Chapman, N. H., Greenwood, C. M. T., and Darlington, G. A. 1995. Evaluation of genetic and environmental effects using GEE and APM methods, Genet. Epidemiol. 12(6), 729-734.

Campbell, D. T., and Stanley, J. C. 1966. "Experimental and QuasiExperimental Designs for Research," Rand McNally, Chicago.

Chakravarti, A. 1998. It's raining SNPs, hallelujah, Nat. Genet. 19, 216-217.

Cohen, J. 1983. The cost of dichotomization, Appl. Psychol. Measur. 7, 249-253.

Cohen, A., and Rom, M. 1994. A method for hypothesis tests in polychotomous logistic-regression, Comput. Stat. Data Anal. 17(3), 277-288.

Curtis, D. 1997. Use of siblings as controls in case-control association studies, Ann. Hum. Genet. 61, 319-333.

Curtis, D., and Sham, P. C. 1995. A note on the application of the transmission disequilibrium test when a parent is missing, Am. J. Hum. Genet. 56, 811-812.

Curtis, D., Miller, M. B., and Sham, P. C. 1999. Combining the sibling disequilibrium test and transmission disequilibrium test for multiallelic markers, Am. J. Hum. Genet. 64, 1785-1786.

Devlin, B., and Roeder, K. 1999. Genomic control for association studies, Biometrics 55, 997-1004.

Dhar, M., Webb, L. S., Smith, L., Hauser, L., Johnson, D., and West, D. B. 2000. A novel ATPase on mouse chromosome 7 is a candidate gene for increased body fat, Physiol. Genomics 4(1), 93-100.

Dolan, C. V., Molenaar, P. C. M., and Boomsma, D. I. 1989. LISREL analysis of twin data with structured means, Behav. Genet. 19, $51-62$.

Dolan, C. V., Boomsma, D. I., and Neale, M. C. 1999. A simulation study of the effects of assignment of prior identity-by-descent probabilities to unselected sib pairs, in covariance-structure modeling of a quantitative trait locus, Am. J. Hum. Genet. 64, 268-280.

Elston, R. C. 1998. Linkage and association, Genet. Epidemiol. 15(6), 565-576. 
Elston, R. C. 2000. Introduction and overview, Stat. Methods Med. Res. 9, 527-541.

Ewens, W. J., and Spielman, R. S. 1995. The transmission/disequilibrium test: History, subdivision, and admixture, Am. J. Hum. Genet. 57, 455-464.

Forrest, W. F., and Feingold, E. 2000. Composite statistics for QTL mapping with moderately discordant sibling pairs, Am. J. Hum. Genet. 66, 1642-1660.

Frankel, W. N., and Schork, N. J. 1996. Who's afraid of epistasis? Nat. Genet. 14(4), 371-373.

Fulker, D. W., and Cherny, S. S. 1996. An improved multipoint sib-pair analysis of quantitative traits, Behav. Genet. 26, 527-532.

Fulker, D. W., Cherny, S. S., Sham, P. C., and Hewitt, J. K. 1999. Combined linkage and association sib pair analysis for quantitative traits, Am. J. Hum. Genet. 64, 259-267.

George, V., Tiwari, H. K., Shu, Y. Y., Zhu, X. F., and Elston, R. C. 1999a. Linkage and association analyses of alcoholism using a regression-based transmission/disequilibrium test, Genet. Epidemiol. 17, S157-S161.

George, V., Tiwari, H. K., Zhu, X. F., and Elston, R. C. 1999b. A test of transmission disequilibrium for quantitative traits in pedigree data, by multiple regression, Am. J. Hum. Genet. 65, 236-245.

Georges, M., and Cockett, N. 1996. The ovine callipyge locus: A paradigm illustrating the importance of non-Mendelian genetics in livestock, Reprod. Nutr. Dev. 36(6), 651-657.

Goddard, M. E. 1992. A mixed model for analyses of data on multiple genetic-markers, Theor. Appl. Genet. 83(6-7), 878-886.

Goldgar, D. E., and Easton, D. F. 1997. Optimal strategies for mapping complex diseases in the presence of multiple loci, Am. J. Hum. Genet. 60, 1222-1232.

GunayAygun, M., Cassidy, S. B., and Nicholls, R. D. 1997. PraderWilli and other syndromes associated with obesity and mental retardation, Behav. Genet. 27(4), 307-324.

Hastbacka, J., de la Chapelle, A., Kaitila, I., Sistonen, P., Weaver, A., and Lander, E. 1992. Linkage disequilibrium mapping in isolated founder populations: Diastrophic dysplasia in Finland, Nat. Genet. 2, 204-211.

Heo, M., Leibel, R. L., Boyer, B. B., Chung, W. K., Koulu, M., Karvonen, M. K., Pesonen, U., Rissanen, A., Laakso, M., Uusitupa, M. I. J., Chagnon, Y., Bouchard, C., Donohoue, P. A., Burns, T. L., Shuldiner, A. R., Silver, K., Andersen, R. E., Pedersen, O., Echwald, S., Sørensen, T. I. A., Behn, P., Permutt, M. A., Jacobs, K. B., Elston, R. C., Hoffman, D. J., and Allison, D. B. 2001. Pooling analysis of genetic data: the association of leptin receptor (LEPR) polymorphisms with variables related to human adiposity, Genetics, in press.

Horvath, S., and Laird, N. M. 1998. A discordant- sibship test for disequilibrium and linkage: No need for parental data, Am. J. Hum. Genet. 63, 1886-1897.

Horvath, S., Laird, N. M., and Knapp, M. 2000. The transmission/ disequilibrium test and parental-genotype reconstruction for X-chromosomal markers, Am. J. Hum. Genet. 66, 1161-1167.

Horvath, S., Xu, X., and Laird, N. M. 2001. The family based association test method: Strategies for studying general genotype-phenotype associations, Eur. J. Hum. Genet. 9, 301-306.

Kaplan, N., and Morris, R. 2001. Issues concerning association studies for fine mapping a susceptibility gene for a complex disease, Genet. Epidemiol. 20(4), 432-457.

Kaplan, N. L., Martin, E. R., and Weir, B. S. 1997. Power studies for the transmission/disequilibrium tests with multiple alleles, $\mathrm{Am}$. J. Hum. Genet. 60, 691-702.
Knapp, M. 1999. The transmission/disequilibrium test and parentalgenotype reconstruction: The reconstruction-combined transmission/disequilibrium test, Am. J. Hum. Genet. 64, 861-870.

Laird, N. M., Horvath, S., and Xu, X. 2000. Implementing a unified approach to family-based tests of association, Genet. Epidemiol. 19, S36-S42.

Lake, S. L., Blacker, D., and Laird, N. M. 2000. Family-based tests of association in the presence of linkage, Am. J. Hum. Genet. 67, $1515-1525$.

Lander, E. S., and Schork, N. J. 1994. Genetic dissection of complex traits, Science 265 (5181), 2037-2048.

Little, R. J. A., and Rubin, D. B. 1987. "Statistical Analysis with Missing Data," Whiley, New York.

Long, A. D., and Langley, C. H. 1999. The power of association studies to detect the contribution of candidate genetic loci to variation in complex traits, Genome Res. 9, 720-731.

Lynch, M., and Walsh, B. 1997. "Genetics and Analysis of Quantitative Traits," Sinauer, Sunderland, MA.

Mangin, B., Thoquet, P., and Grimsley, N. 1998. Pleiotropic QTL analysis, Biometrics 54, 88-99.

Marinov, M., and Weeks, D. E. 2001. The complexity of linkage analysis with neural networks, Hum. Hered. 51, 169-176.

McNemar, Q. 1947. Note on the sampling error of the difference between correlated proportions or percentages, Psychometrika 12, 153-157.

Mokliatchouk, O., Blacker, D., and Rabinowitz, D. 2000. Association tests for traits with variable age at onset, Hum. Hered. 51, 46-53.

Morley, J. E., Baumgartner, R. N., Roubenoff, R., Mayer, J., and Nair, K. S. 2001. Sarcopenia, J. Lab. Clin. Med. 137, 231-243.

Morton, N. E., and Collins, A. 1998. Tests and estimates of allelic association in complex inheritance, Proc. Natl. Acad. Sci. USA 95, 11389-11393.

Neale, M. C. 2001. The use of Mx for association and linkage analysis, Genescreen 1, 107-112.

Neale, M. C., Cherny, S. S., Sham, P. C., Whitfield, J. B., Heath, A. C., Birley, A. J., and Martin, N. G. 1999. Distinguishing population stratification from genuine allelic effects with $\mathrm{Mx}$ : Association of ADH2 with alcohol consumption, Behav. Genet. 29, 233-243.

Neter, J., Wasserman, W., and Kutner, M. H. 1990. "Applied Linear Statistical Models,' Irwin, Boston.

Perusse, L., Chagnon, Y. C., Weisnagel, S. J., Rankinen, T., Snyder, E., Sands, J., and Bouchard, C. 2001. The human obesity gene map: The 2000 update, Obes. Res. 9(2), 135-169.

Pritchard, J. K., and Rosenberg, N. A. 1999. Use of unlinked genetic markers to detect population stratification in association studies, Am. J. Hum. Genet. 65, 220-228.

Pritchard, J. K., Stephens, M., Rosenberg, N. A., and Donnelly, P. 2000a. Association mapping in structured populations, Am. J. Hum. Genet. 67, 170-181.

Pritchard, J. K., Stephens, M., and Donnelly, P. 2000b. Inference of population structure using multilocus genotype data, Genetics 155 , 945-959.

Rabinowitz, D. 1997. A transmission/disequilibrium test for quantitative trait loci, Hum. Hered. 47, 342-350.

Rabinowitz, D., and Laird, N. 2000. A unified approach to adjusting association tests for population admixture with arbitrary pedigree structure and arbitrary missing marker information, Hum. Hered. 50, 211-223.

Reich, D. E., and Goldstein, D. B. 2001. Detecting association in a case-control study while correcting for population stratification, Genet. Epidemiol. 20, 4-16. 
Robinson, S. W., Dinulescu, D. M., and Cone, R. D. 2000. Genetic models of obesity and energy balance in the mouse, Annu. Rev. Genet. 34, 687-745.

Rosenbaum, P. R., and Rubin, D. B. 1984. Reducing bias in observational studies using subclassification on the propensity score, J. Am. Stat. Assoc. 79(387), 516-524.

Rubin, D. B. 1991. Practical implications of modes of statistical-inference for causal effects and the critical role of the assignment mechanism, Biometrics 47(4), 1213-1234.

Satten, G. A., Flanders, W. D., and Yang, Q. H. 2001. Accounting for unmeasured population substructure in case-control studies of genetic association using a novel latent-class model, Am. J. Hum. Genet. 68, $466-477$.

Schafer, J. L. 1997. "Analysis of Incomplete Multivariate Analysis. Monographs on Statistics and Applied Probability," Series 72, Chapman \& Hall, New York.

Schaid, D. J., and Li, H. Z. 1997. Genotype relative-risks and association tests for nuclear families with missing parental data, Genet. Epidemiol. 14, 1113-1118.

Schaid, D. J., and Rowland C. 1998. Use of parents, sibs and unrelated controls for detection of associations between genetic markers and disease, Am. J. Hum. Genet. 63, 1492-1506.

Schaid, D. J., and Rowland, C. M. 1999. Quantitative trait transmission disequilibrium test: Allowance for missing parents, Genet. Epidemiol. 17, S307-S312.

Schaid, D. J., and Rowland, C. M. 2000. Robust transmission regression models for linkage and association, Genet. Epidemiol. 19, S78-S84.

Sen, A., and Srivastava, M. 1990. "Regression Analysis: Theory, Methods, and Applications," Springer-Verlag, New York.

Simpson, E. H. 1951. The interpretation of interaction in contingency tables, J. R. Statist. Soc. B 13, 238-241.

Sorbom, D. 1974. A general method for studying differences in factor means and factor structures between groups, Br. J. Math. Stat. Psychol. 27, 229-239.

Spielman, R. S., McGinnis, R. E., and Ewens, W. J. 1993. Transmission test for linkage disequilibrium: The insulin gene region and insulin-dependent diabetes mellitus (IDDM), Am. J. Hum. Genet. 52, 506-516.

Spielman, R. S., and Ewens, W. J. 1998. A sibship test for linkage in the presence of association: The sib transmission/disequilibrium test, Am. J. Hum. Genet. 62, 450-458.

Sun, F. Z., Flanders, W. D., Yang, Q. H., and Zhao, H. Y. 2000. Transmission/disequilibrium tests for quantitative traits, Ann. Hum. Genet. 64, 555-565.

van den Oord, E. J. C. G. 2000. The use of mixture models to perform quantitative tests for linkage disequilibrium, maternal effects, and parent-of origin effects with incomplete subject-parent triads, Behav. Genet. 30, 335-343.

Waldman, I. D., Robinson, B. F., and Rowe, D. C. 1999. A logistic regression based extension of the TDT for continuous and categorical traits, Ann. Hum. Genet. 63, 329-340.

Weinberg, C. R. 1999. Allowing for missing parents in genetic studies of case-parent triads, Am. J. Hum. Genet. 64, 1186-1193.

Whittemore, A. S., and Tu, I. P. 2000. Detection of disease genes by use of family data. I. Likelihood-based theory, Am. J. Hum. Genet. 66, 1328-1340.

Woodward, J. A., Bonett, D. G., and Brecht, M. L. 1990. "Introduction to Linear Models and Experimental Design," Harcourt Brace Jovanovich, New York.

Yasue, H., Mikawa, S., Uenishi, H., and Wada, Y. 1999. Analysis of allele segregation distortion in a swine resource family, Anim. Biotechnol. 10(3), 147-152.

Zhao, H. Y. 2000. Family-based association studies, Stat. Methods Med. Res. 9(6), 563-587.

Zhao, H., and Sun, F. 1999. On a unified transmission/disequilibrium test, Am. J. Hum. Genet. 65, 2583.

Zhu, X. F., Elston, R. C., and Cooper, R. S. 2001. Testing quantitative traits for association and linkage in the presence or absence of parental data, Hum. Hered. 51, 183-191.

Zhu, X. F., and Elston, R. C. 2000. Power comparison of regression methods to test quantitative traits for association and linkage, Genet. Epidemiol. 18, 322-330. 